\title{
Kerov functions revisited
}

\author{
A.Mironov $^{a, b, c *}$ and A.Morozov ${ }^{b, c \dagger}$ \\ ${ }^{a}$ Lebedev Physics Institute, Moscow 119991, Russia \\ ${ }^{b}$ ITEP, Moscow 117218, Russia \\ ${ }^{c}$ Institute for Information Transmission Problems, Moscow 127994, Russia
}

\begin{abstract}
The Schur functions play a crucial role in the modern description of HOMFLY polynomials for knots and of topological vertices in DIM-based network theories, which could merge into a unified theory still to be developed. The Macdonald functions do the same for hyperpolynomials and refined vertices, but merging appears to be more problematic. For a detailed study of this problem, more knowledge is needed about the Macdonald polynomials than is usually available. As a preparation for the discussion of the knot/vertices relation, we summarize the relevant facts and open problems about the Macdonald and, more generally, Kerov functions. Like Macdonald polynomials, they are triangular combinations of Schur functions, but orthogonal in a more general scalar product. We explain that parameters of the measure can be considered as a set of new time variables, and the Kerov functions are actually expressed through the Schur functions of these variables as well. Despite they provide an infinite-parametric extension of the Schur and Macdonald polynomials, the Kerov functions, and even the skew Kerov functions continue to satisfy the most important relations, like Cauchy summation formula, the transposition identity for reflection of the Young diagram and expression of the skew functions through the generalized Littlewood-Richardson structure constants. Since these are the properties important in most applications, one can expect that the Kerov extension exists for most of them, from the superintegrable matrix and tensor models to knot theory.
\end{abstract}

\section{Introduction}

Recently in [1] a program was originated to construct various (not obligatory torus) link polynomials [2] from topological vertices [3. Already in the simplest example of the link $L_{8 n 8}$ considered in that paper, one encounters composite representations, associated "uniform" Schur functions and their decompositions into bilinear combinations of the more conventional skew Schur functions, and the skew Schur is what is actually associated with the topological vertex.

This technique is however not easily lifted from the HOMFLY polynomials to the level of hyperpolynomials (the algebraically defined counterparts of Khovanov-Rozansky and superpolynomials), largely because of our insufficient knowledge about the Macdonald polynomials, which substitute the Schur functions in this lifting. This paper is a preparation for developing a theory of composite Macdonald functions and its application to study of the hyperpolynomial/refined-vertex relation, which will be presented elsewhere 4. Needed for this theory are the basic facts about the Macdonald functions in the form which allows one to easily calculate them for arbitrary representations $R$ at sufficiently high levels $|R|$.

In this paper, we make such a list of properties, moreover, we present it for more general Kerov functions [5], because in applications it is important to understand what in these functions is depending on the obstacles for the Schur $\longrightarrow$ Macdonald $\longrightarrow$ Kerov extension, what are the ways to overcome these obstacles and what one needs to sacrifice when doing this.

We begin with the most general Kerov functions and then descend to the Macdonald and Schur functions, which have some additional properties absent in the Kerov case. The very few properties, which are true only for the Schur functions and are destroyed already by the Macdonald deformation are underlined, those which are true for the Macdonald, but not for generic Kerov functions are underlined twice. The Macdonald functions have closer relation to group theory but many of their properties are actually true in the more general Kerov framework.

The Kerov function should not be confused with much better known "Kerov character polynomials", also associated with the name of S.Kerov [7]: to avoid the confusion, we use the term functions throughout the present text.

\footnotetext{
*mironov@lpi.ru; mironov@itep.ru

†morozov@itep.ru
} 


\section{Kerov functions and their properties}

- The pair of Kerov functions $\operatorname{Ker}_{R}^{(g)}\{p\}$ and $\widehat{\operatorname{Ker}}_{R}^{(g)}\{p\}$ depends on the Young diagram (representation) $R=\left[r_{1} \geq r_{2} \geq \ldots \geq r_{l_{R}}>0\right]$, they are polynomials of infinitely many time-variables $p_{k}$ homogeneous w.r.t. the grading $\operatorname{grad}\left(p_{k}\right)=k$. Thus, a particular $\operatorname{Ker}_{R}\{p\}$ depends only on $|R|$ times, the level $|R|$ being the size of $R$ (number of boxes). The two functions $\operatorname{Ker}_{R}^{(g)}\{p\}, \widehat{\operatorname{Ker}}_{R}^{(g)}\{p\}$ differ by the choice of ordering in the sums in (1) below.

- The best for practical purposes is to define the Kerov and Macdonald functions by a triangular transform from the Schur functions,

$$
\left\{\begin{array}{c}
\operatorname{Ker}_{R}^{(g)}\{p\}=\operatorname{Schur}_{R}\{p\}+\sum_{R^{\prime}<R} \mathcal{K}_{R, R^{\prime}}^{(g)} \cdot \operatorname{Schur}_{R^{\prime}}\{p\} \\
\widehat{\operatorname{Ker}}_{R}^{(g)}\{p\}=\operatorname{Schur}_{R}\{p\}+\sum_{R^{\prime v}>R^{\vee}} \widehat{\mathcal{K}}_{R^{\prime v}, R^{\vee}}^{(g)} \cdot \operatorname{Schur}_{R^{\prime}}\{p\}
\end{array}\right\}
$$

where the Young diagrams are ordered lexicographically:

$R>R^{\prime}$ if $r_{1}>r_{1}^{\prime}$ or if $r_{1}=r_{1}^{\prime}$, but $r_{2}>r_{2}^{\prime}$, or if $r_{1}=r_{1}^{\prime}$ and $r_{2}=r_{2}^{\prime}$, but $r_{3}>r_{3}^{\prime}$, and so on

The pair of functions emerges because this ordering is not consistent with the transposition of Young diagrams:

$$
R>R^{\prime} \text { is not always the same as } R^{\prime \vee}>R^{\vee}
$$

The first discrepancy appears at level $|R|=6$, it is the pair $[3,1,1,1]>[2,2,2]$, for which $[3,1,1,1]^{\vee}=$ $[4,1,1]>[2,2,2]^{\vee}=[3,3]$.

- A similar definition through the triangular transformation is also most efficient for the generalized Macdonald functions [8], which depend on several Young diagrams and several sets of time-variables. Moreover, it seems also applicable to the plane partition generalizations [9]. In the conventional approach, in all these cases the triangularity is associated with a peculiar (triangular) choice of the Hamiltonian deformations, which appear relevant to the Calogero-Ruijenaars systems and to matrix models (and thus to the AGT relations). One of the possible explanations for this can be through the PQ (spectral) duality, which is the basic semi-hidden symmetry of generic network models.

- The Macdonald-Kostka coefficients $\mathcal{K}_{R, R^{\prime}}^{(g)}$ can be defined iteratively in $R$ and $R^{\prime}$ from the orthogonality conditions

$$
\left\langle\operatorname{Ker}_{R}^{(g)} \mid \operatorname{Ker}_{R^{\prime}}^{(g)}\right\rangle=\left\|\operatorname{Ker}_{R}^{(g)}\right\|^{2} \cdot \delta_{R, R^{\prime}}
$$

with the scalar product given by

$$
\left\langle p^{\Delta} \mid p^{\Delta^{\prime}}\right\rangle^{(g)}=z_{\Delta} \cdot \delta_{\Delta, \Delta^{\prime}} \cdot\left(\prod_{i=1}^{l_{\Delta}} g_{\delta_{i}}\right)
$$

Here the Young diagram $\Delta=\left[\delta_{1} \geq \delta_{2} \geq \ldots \geq \delta_{l_{\Delta}}\right]$, and $p^{\Delta}=\prod_{i=1}^{l_{\Delta}} p_{\delta_{i}}$. The combinatorial factor $z_{\Delta}$ is best defined in the dual parametrization of the Young diagram, $\Delta=\left[\ldots, 2^{m_{2}}, 1^{m_{1}}\right]$, then $z_{\Delta}=$ $\prod_{k} k^{m_{k}} \cdot m_{k}$ !. Note that the normalization of $\operatorname{Ker}^{(g)}$ is already fixed by the choice of unit diagonal coefficient (the first term) in (11):

$$
\mathcal{K}_{R R}^{(g)}=1
$$

therefore the norm $\left\|\operatorname{Ker}^{(g)}\right\|$ is a deducible quantity.

- The Schur functions $\operatorname{Schur}_{R}=\operatorname{Ker}_{R}^{(g=1)}$ are orthonormal at $g=1$ :

$$
\underline{\left\langle\operatorname{Schur}_{R} \mid \operatorname{Schur}_{R^{\prime}}\right\rangle^{(g=1)}=\delta_{R, R^{\prime}}}
$$


The Macdonald-Kostka matrices $\mathcal{K}^{(g)}$ and $\widehat{\mathcal{K}}^{(g)}$ (both!) diagonalize their product matrix with $g \neq 1$,

$$
\mu_{R, R^{\prime}}=\left\langle\operatorname{Schur}_{R} \mid \operatorname{Schur}_{R^{\prime}}\right\rangle^{(g)}=\sum_{\Delta} \frac{\psi_{R_{1}}(\Delta) \psi_{R_{2}}(\Delta)}{z_{\Delta}} \cdot g_{\Delta}
$$

i.e. provide its Gauss decomposition for two different choices of the triangular (Borel) structure. Here $\psi_{R}(\Delta)$ are the symmetric group characters. Note that $g_{\Delta}=\prod_{i=1}^{l_{\Delta}} g_{\delta_{i}}$ appear here in a combination, similar to $p^{\Delta}$ in (5), and this is the first manifestation of a far-going similarity between $g_{n}$ and time-variables, which we will further review in detail in sec 7 below.

The Gauss decomposition is a very efficient operation in computer simulations. An additional advantage of (8) is that the orthogonality of characters $\psi_{R}(\Delta)$ implies an equally simple expression for an arbitrary power of $\mu$ including the inverse matrix $\mu^{-1}$, the one which should actually be decomposed to provide the Kostka matrix $\mathcal{K}^{(g)}$ :

$$
\left(\mu^{-1}\right)_{R, R^{\prime}}=\sum_{\Delta} \frac{\psi_{R_{1}}(\Delta) \psi_{R_{2}}(\Delta)}{z_{\Delta}} \cdot \frac{1}{g_{\Delta}}=\mu_{R, R^{\prime}}^{\left(g^{-1}\right)} \Longleftarrow \quad \Longleftarrow \quad\left(\mu^{n}\right)_{R, R^{\prime}}=\mu_{R, R^{\prime}}^{\left(g^{n}\right)} \quad n \in \mathbb{Z}
$$

The orthogonality conditions (4) can also be used to represent the Macdonald-Kostka coefficients $\mathcal{K}_{R, R^{\prime}}^{(g)}$ in (11) as determinants/minors of the matrix $\mu_{R, R^{\prime}}$.

- Being a complete basis in the space of $p$-polynomials, the Kerov functions satisfy the multiplication rules

$$
\begin{gathered}
\operatorname{Ker}_{R}^{(g)}\{p\} \cdot \operatorname{Ker}_{R^{\prime}}^{(g)}\{p\}=\sum_{R \cup R^{\prime} \leq R^{\prime \prime} \leq R+R^{\prime}} N_{R, R^{\prime}}^{R^{\prime \prime}}(g) \cdot \operatorname{Ker}_{R^{\prime \prime}}^{(g)}\{p\} \\
\widehat{\operatorname{Ker}}_{R}^{(g)}\{p\} \cdot \widehat{\operatorname{Ker}}_{R^{\prime}}^{(g)}\{p\}=\sum_{R^{\vee}+R^{\prime \vee} \leq R^{\prime \prime} \leq R^{\vee} \cup R^{\prime \vee}} \widehat{N}_{R, R^{\prime}}^{R^{\prime \prime}}(g) \cdot \widehat{\operatorname{Ker}}_{R^{\prime \prime}}^{(g)}\{p\} \\
\hline
\end{gathered}
$$

and the sums are rather restricted, since the generalized Littlewood-Richardson coefficients $N_{R, R^{\prime}}^{R^{\prime \prime}}(g)$ are non-zero only in between the partitions $R \cup R^{\prime}=\left[r_{1}+r_{1}^{\prime}, r_{2}+r_{2}^{\prime}, \ldots\right]$ and $R+R^{\prime}=$ [ordered collection of all $r_{i}$ and $\left.r_{j}^{\prime}\right]$.

- Also obviously existing is the decomposition

$$
\operatorname{Ker}_{R}^{(g)}\left\{p+p^{\prime}\right\}=\sum_{R^{\prime} \subset R} \operatorname{Ker}_{R^{\prime}}^{(g)}\{p\} \cdot \operatorname{Ker}_{R / R^{\prime}}^{(g)}\left\{p^{\prime}\right\}
$$

which defines the skew Kerov functions, and it is actually a dual of (10), because (not quite trivially)

$$
\operatorname{Ker}_{R / R^{\prime}}^{(g)}\{p\}=\sum_{R^{\prime \prime}} \widehat{N}_{R^{\prime \vee} R^{\prime \prime}}^{R^{\vee}}\left(g^{-1}\right) \cdot \operatorname{Ker}_{R^{\prime \prime}}^{(g)}\{p\}
$$

with the same $N$ as in (10).

- Equivalence of the two latter decompositions is consistent with the transposition rule

$$
\begin{gathered}
\operatorname{Ker}_{R}^{(g)}\{p\}=(-)^{|R|} \cdot\left\|\operatorname{Ker}_{R}^{(g)}\right\|^{2} \cdot \widehat{\operatorname{Ker}}_{R^{\vee}}^{\left(g^{-1}\right)}\left\{-p^{\vee}\right\} \\
\mathbb{} \\
\operatorname{Ker}_{R}^{(g)}\left\{p^{\wedge}\right\}=(-)^{|R|} \cdot\left\|\operatorname{Ker}_{R}^{(g)}\right\|^{2} \cdot \widehat{\operatorname{Ker}}_{R^{\vee}}^{\left(g^{-1}\right)}\{-p\}
\end{gathered}
$$

where operation ${ }^{\wedge}$ on the time-variables is inverse of $\vee^{\vee}:\left(p^{\wedge}\right)^{\vee}=p$, and, explicitly,

$$
p_{k}^{\vee}=\frac{p_{k}}{g_{k}} \quad \text { and } \quad p_{k}^{\wedge}=g_{k} \cdot p_{k}
$$


- One can also introduce a dual Kerov function (in complete analogy with the Macdonald case, [10]):

$$
\underline{\operatorname{Ker}}_{R}^{(g)}\{p\}:=\frac{\operatorname{Ker}_{R}^{(g)}\{p\}}{\left\|\operatorname{Ker}_{R}^{(g)}\right\|^{2}}
$$

With this definition, formula (13) looks a bit simpler

$$
\underline{\operatorname{Ker}}_{R}^{(g)}\{p\}=(-)^{|R|} \widehat{\operatorname{Ker}}_{R^{\vee}}^{\left(g^{-1}\right)}\left\{-p^{\vee}\right\}
$$

Moreover, introducing the corresponding dual skew Kerov functions,

$$
\underline{\operatorname{Ker}}_{R}^{(g)}\left\{p+p^{\prime}\right\}=\sum_{R^{\prime} \subset R} \underline{\operatorname{Ker}}_{R^{\prime}}^{(g)}\{p\} \cdot \underline{\operatorname{Ker}}_{R / R^{\prime}}^{(g)}\left\{p^{\prime}\right\}
$$

one obtains

$$
\underline{\operatorname{Ker}}_{R / R^{\prime}}^{(g)}\{p\}=\sum_{R^{\prime \prime}} N_{R^{\prime} R^{\prime \prime}}^{R}(g) \cdot \underline{\operatorname{Ker}}_{R^{\prime \prime}}^{(g)}\{p\}
$$

- Important in applications is the Cauchy summation formula:

$$
\sum_{R}(-)^{|R|} \operatorname{Ker}_{R}^{(g)}\{p\} \cdot \widehat{\operatorname{Ker}}_{R^{\vee}}^{\left(g^{-1}\right)}\left\{-p^{\prime}\right\}=\sum_{R} \frac{\operatorname{Ker}_{R}^{(g)}\{p\} \cdot \operatorname{Ker}_{R}^{(g)}\left\{p^{\prime \wedge}\right\}}{\left\|\operatorname{Ker}_{R}^{(g)}\right\|^{2}}=\exp \left(\sum_{k} \frac{p_{k} p_{k}^{\prime}}{k}\right)
$$

and, more generally,

$$
\sum_{R}(-)^{|R|} \operatorname{Ker}_{R / \eta}^{(g)}\{p\} \cdot \widehat{\operatorname{Ker}}_{R^{\vee} / \zeta}^{\left(g^{-1}\right)}\left\{-p^{\prime}\right\}=\exp \left(\sum_{k} \frac{p_{k} p_{k}^{\prime}}{k}\right) \cdot \sum_{\sigma}(-)^{|\zeta|+|\eta|+|\sigma|} \operatorname{Ker}_{\zeta^{\vee} / \sigma}^{(g)}\{p\} \cdot \widehat{\operatorname{Ker}}_{\eta^{\vee} / \sigma^{\vee}}^{\left(g^{-1}\right)}\left\{-p^{\prime}\right\}
$$

where the sum over $\sigma$ at the r.h.s. contains only finitely many terms.

\section{Specialization of the function $g$}

- In the FZ parametrization [11 motivated by applications to the XYZ model (at $\kappa=2$ ), the function $g_{n}$ depends on several $q$ and $t$ parameters:

$$
g_{n}=\prod_{\alpha=1}^{\kappa} \frac{\left\{q_{\alpha}^{n}\right\}}{\left\{t_{\alpha}^{n}\right\}}
$$

where $\{x\}=x-x^{-1}$. For the Schur functions, $\underline{\kappa=0}$, for the Macdonald functions, $\underline{\underline{\kappa=1}}$. Thus, the Schur functions are orthogonal in the product

$$
\underline{\left\langle p^{\Delta} \mid p^{\Delta^{\prime}}\right\rangle^{(1)}=z_{\Delta} \cdot \delta_{\Delta, \Delta^{\prime}}}
$$

and their norm is $\left\|\operatorname{Ker}_{R}^{(\kappa=0)}\right\|^{2}=1$, while the Macdonald functions $\operatorname{Mac}\{q, t, p\}$, are orthogonal in

$$
\underline{\left\langle p^{\Delta} \mid p^{\Delta^{\prime}}\right\rangle^{(\mathrm{Mac})}=z_{\Delta} \cdot \delta_{\Delta, \Delta^{\prime}} \cdot \prod_{i=1}^{l_{\Delta}} \frac{\left\{q_{i}^{\delta}\right\}}{\left\{t^{\delta_{i}}\right\}}}
$$

and their norm is

$$
\underline{\underline{ }} \underline{\operatorname{Ker}_{R}^{(\kappa=1)} \|^{2}=\frac{h_{R^{\vee}}(t, q)}{h_{R}(q, t)}}
$$

where

$$
h_{R}(q, t):=\prod_{i, j \in R}\left(q^{j-R_{i}} t^{i-R_{j}^{\vee}+1}-q^{R_{i}-j} t^{R_{j}^{\vee}-i-1}\right)
$$


- If $g_{n}$ are considered as additional time-variables, Macdonald locus $\underline{\underline{g_{n}=\frac{\left\{q^{n}\right\}}{\left\{t^{n}\right\}}}}$ becomes a direct counterpart of the topological locus [12] $p_{k}=\frac{\left\{A^{k}\right\}}{\left\{t^{k}\right\}}$ for the ordinary time-variables. This analogy nicely explains both the distinguished role of Macdonald case in many circumstances, as well as its limitations and the need of further extension to the full-fledged Kerov case with arbitrary $g_{n}$. The main peculiarity of topological locus are nice factorization properties, but in the case of, say, knot theory, they are directly helpful in evolution formulas [13] and in differential expansions [14, while some equally important properties like integrability [15] in torus links and knots [12, 16, 18, are available only if one looks from beyond the topological locus [19. What really matters is superintegrability, it is reflected in factorization at the topological/Macdonald loci, but is actually a far more general property of the Schur functions and their relatives, which holds far beyond these small 2-dimensional varieties in the infinite-dimensional space of time-variables, and is, perhaps, extendable to the entire world of Kerov functions. As we suggest in sec7 below, it can be not only extendable, but becomes much richer after this extension is done: the symmetries are not really lost, but rather enhanced.

\section{Relation to symmetric polynomials and to representation theory}

- On the $n$-dimensional Miwa locus in the time-variable space,

$$
p_{k}=\operatorname{tr} X^{k}=\sum_{a=1}^{n} x_{a}^{n}
$$

the function $\operatorname{Ker}_{R}$ turns into a symmetric polynomial

$$
\operatorname{Ker}_{R}[X]=\sum_{R^{\prime}<R} k_{R, R^{\prime}}^{(g)} \cdot m_{R^{\prime}}[X]
$$

which is a triangular sum of monomial symmetric polynomials

$$
m_{R}[X]=\operatorname{symm}_{x}\left(\prod_{a=1}^{l_{R}} x_{a}^{r_{a}}\right)
$$

- The Kostka numbers

$$
\underline{k_{R, R^{\prime}}^{\text {(Schur) }}=K_{R, R^{\prime}}^{-1}(q=0, t=1)}
$$

are the values of inverse Macdonald-Kostka matrix $K$ at the particular values of parameters $q=0$ and $t=1$ (inverted is the matrix, not the particular entries). This is the original Macdonald's requirement that, at these values, his polynomials coincide with $m_{R}: M_{R}[0,1, X]=m_{R}[X]$.

- Conceptually, the Schur functions are symmetric polynomials associated with representation theory of $G L_{n}$ (generalizations to other root systems also exist). They are defined so that $\operatorname{Schur}[X]$ for $n=\operatorname{rank}\left(G L_{n}\right)$ are the characters (elements of the center of the group algebra) of $G L_{n}$. As a corollary, the sum in multiplication rule (10) is now restricted to

$$
\underline{\underline{R^{\prime \prime} \in R \otimes R^{\prime}}}
$$

In the Kerov case, the sum includes contributions not appearing in the product of representations. For the first time, this happens at the level $|R|$, for example,

$$
\operatorname{Ker}_{[4]} \cdot \operatorname{Ker}_{[1,1]}=\alpha \cdot \operatorname{Ker}_{[4,1,1]}+\beta \cdot \operatorname{Ker}_{[4,2]}+\gamma \cdot \operatorname{Ker}_{[5,1]}
$$

while $[4] \otimes[1,1]=[4,1,1]+[5,1]$. Moreover, for the $\widehat{K}$-Kerov functions, the same relation contains not three, but four terms:

$$
\operatorname{Ker}_{[4]} \cdot \operatorname{Ker}_{[1,1]}=\hat{\alpha} \cdot \widehat{\operatorname{Ker}}_{[4,1,1]}+\hat{\beta} \cdot \operatorname{Ker}_{[4,2]}+\hat{\delta} \cdot \widehat{\operatorname{Ker}}_{[3,3]}+\hat{\gamma} \cdot \operatorname{Ker}_{[5,1]}
$$

because there are two diagrams $[2,2,1,1]=[4,2]^{\vee}$ and $[2,2,2]=[3,3]^{\vee}$ between $[2,1,1,1,1]=[5,1]^{\vee}$ and $[3,1,1,1]=[4,1,1]^{\vee}$. We made it explicit that only two of the four emerging $\widehat{K}$-Kerov functions are different from the $K$-ones. 
- In the Macdonald case, however, (30) is believed to survive. In particular, the coefficient $\beta$ in (31), vanishes in the Schur and Macdonald cases $\kappa=0,1$, but not for $\kappa>1$.

As a related (but not equivalent) property, on the three-dimensional locus $g(k)=x_{1}^{k}+x_{2}^{k}+x_{3}^{k}$

$$
\beta \sim x_{1}^{2} x_{2}^{2} x_{3}^{2}\left(x_{1}^{2}-x_{2} x_{3}\right)\left(x_{2}^{2}-x_{1} x_{3}\right)\left(x_{3}^{2}-x_{1} x_{2}\right) \cdot B(x)
$$

with a complicated irreducible polynomial $B(x)$. There is also a relatively simple denominator.

\section{Equivalence of $\widehat{\mathrm{Ker}}$ and Ker in Macdonald case}

Actually only half of the Macdonald functions needs to be calculated directly, the other half can be obtained from (13). This is a big calculational advantage, because the computer time needed for orthogonalization grows dramatically with the number of terms in the sum (11), and reducing the problem to sums of twice smaller length allows one to calculate the Kerov/Macdonald functions for higher levels $|R|$.

At the same, for the Kerov functions this is not quite correct because of the two types of the Kerov functions related by (13). In other words, (13) is a duality, and not self-duality condition in the generic Kerov case.

- It is remarkable that (13) is a self-duality condition not only in the Schur, but also in the Macdonald case of $\kappa=1$ when it is sufficient to define $\underline{\underline{\mathrm{Mac}}=\mathrm{Mac}}$ so that (13) becomes

$$
\underline{\underline{\operatorname{Mac}_{R}\left\{q, t, p_{k}\right\}=(-)^{|R|} \cdot\left\|\operatorname{Mac}_{R}\right\|^{2} \cdot \operatorname{Mac}_{R^{\vee}}\left\{t, q,-\frac{\left\{t^{k}\right\}}{\left\{q^{k}\right\}} p_{k}\right\}}}
$$

In the Schur case, this further simplifies to just

$$
\operatorname{Schur}_{R^{\vee}}\{p\}=(-)^{|R|} \cdot \operatorname{Schur}_{R}\{-p\}
$$

- The reason for this simplification is that, at $\kappa=1$, all the problematic Macdonald-Kostka coefficients, i.e. those for the pairs of diagrams, when both $R>R^{\prime}$ and $R^{\vee}>R^{\prime}$, are vanishing, for example

$$
\underline{\mathcal{K}_{[3,1,1,1],[2,2,2]}^{(\mathrm{Mac})}=0}
$$

This vanishing condition imposes severe restrictions on the function $g$, which are satisfied for $\kappa=1$, i.e. for the Macdonald deformation, but are violated for $\kappa>1$, i.e. for the generic Kerov functions.

The analogue of (33) is also true: on the 3-dimensional locus $g(k)=x_{1}^{k}+x_{2}^{k}+x_{3}^{k}$ this Macdonald-Kostka coefficient reduces to

$$
\mathcal{K}_{[3,1,1,1],[2,2,2]} \sim x_{1}^{2} x_{2}^{2} x_{3}^{2}\left(x_{1}^{2}-x_{2} x_{3}\right)\left(x_{2}^{2}-x_{1} x_{3}\right)\left(x_{3}^{2}-x_{1} x_{2}\right) \cdot \operatorname{Schur}_{[7]}\{g\}
$$

but the substitute of the polynomial $B(x)$ in this case is actually much simpler. Surprisingly, one again arrives at the Macdonald properties of the group theory expansion (30) and self-duality (34) on the twodimensional locus, where both $\beta$ in (33) and $\mathcal{K}_{[3,1,1,1],[2,2,2]}$ in (37) vanish. It can be chosen, for instance, at $x_{3}^{2}=x_{1} x_{2}$.

- Actually, in the Schur and Macdonald cases, one can use in (10) any partial ordering of Young diagrams which satisfies the dominance rule:

$$
\underline{R \geq R^{\prime} \text { if } \sum_{a=1}^{i} r_{a} \geq \sum_{a=1}^{i} r_{a}^{\prime} \text { for all } i}
$$

since, for all pairs which remain unordered, the corresponding Kostka numbers vanish, together with their inverse, e.g.

$$
\underline{\underline{k_{[3,1,1,1],[2,2,2]}^{ \pm 1}=0}}
$$

(inversion here, as in similar cases before, is inversion of the entire triangular matrix, not of the particular entry).

To finish this piece of the story, we emphasize once again that the duality (13) is rather non-trivial, and it guarantees that the Kerov/Macdonald functions for symmetric representations and "nearly-symmetric" are actually as simple as those for the antisymmetric, i.e. equivalence of the symmetric and antisymmetric "ends" of the partition list, which is far from obvious from the definition (1), where the antisymmetric functions coincide with the antisymmetric Schur polynomials, while symmetric are combinations of all Schur functions at a given level. 


\section{Properties peculiar for the Schur functions}

- Specific for the Schur functions per se is determinant expression through those in symmetric representations

$$
\underline{S_{R}=\operatorname{det}_{i, j=1}^{l_{R}} S_{\left[r_{i}-i+j\right]}, \quad S_{[<0]}=0}
$$

Deformations of this formula exist, for example, one can treat in this way the determinant formula for the Macdonald-Kostka coefficients.

- The Schur functions are expanded in symmetric-group characters $\psi_{R}(\Delta)$ :

$$
\operatorname{Schur}_{R}\{p\}=\sum_{\Delta} \frac{\psi_{R}(\Delta)}{z_{\Delta}} \cdot p^{\Delta}
$$

and their orthogonality (7) is related to the orthogonality of characters,

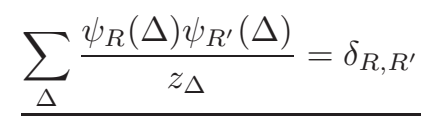

In both these formulas, the sums are over Young diagrams of size $|\Delta|=|R|=\left|R^{\prime}\right|$. Possible Kerov and Macdonald deformations of (41) would require a deep understanding of the Schur-Weyl-Howe duality [20] and is related 21] to deep and yet unanswered questions about the DIM algebra 22] and its further generalizations.

- As described in detail in [23, the Schur functions are the common eigenfunctions of the infinite set of commuting generalized cut-and-join operators $\hat{W}_{\Delta}$ with eigenvalues made from symmetric group characters $\psi_{R}(\Delta)$ :

$$
\underline{\hat{W}_{\Delta}\left\{p_{k}, \frac{\partial}{\partial p_{k}}\right\} \operatorname{Schur}_{R}\{p\}=\frac{\psi_{R}(\Delta)}{d_{R} z_{\Delta}} \cdot \operatorname{Schur}_{R}\{p\}}
$$

where $d_{R}:=\operatorname{Schur}_{R}\left\{\delta_{k, 1}\right\}$. Existence of such operators at each level is a trivial linear-algebra property easily generalizable to the Macdonald and Kerov functions (as well as far beyond them, see, for example, 9]). However, in the Schur (and, partly, Macdonald) case, they can be "unified" into an operator made by the Sugawara construction 24] from a linear Cherednik-Dunkl operator $\hat{J}$ (a counterpart of the KacMoody current in the WZW model [25]): $\hat{W}_{R} \sim \operatorname{Tr} \hat{J}^{R}$, which interrelates naive operators at different levels. Getting a deeper understanding of these relations and their proper extension beyond the Schur case is one of the many open questions in the field.

- The Gaussian averages of the Schur functions provide the same functions evaluated at the topological locus [26]:

$$
\begin{array}{r}
\frac{\int_{n \times n} \operatorname{Schur}_{R}[X] e^{-\operatorname{tr} X^{2}} d X \sim \operatorname{Schur}_{R}\left\{p_{k}=N\right\}=\operatorname{dim}_{N}(R)}{\int_{n \times n} \operatorname{Schur}_{R}\left[e^{X}\right] e^{-\frac{1}{\hbar} \operatorname{tr} X^{2}} d X \sim \operatorname{Schur}_{R}\left\{p_{k}=\frac{\left\{e^{\hbar N}\right\}}{\left\{e^{\hbar}\right\}}\right\}=D_{N}(R)} \\
\hline
\end{array}
$$

which are ordinary and quantum dimensions of the representation $R$ of $S L_{n}$ and $U_{e^{\hbar}}\left(S L_{n}\right)$ correspondingly. This property is responsible for the superintegrability of matrix models [27, it has important generalizations in many directions, from knot theory to a non-trivial extension to tensor models [28. Its Macdonald generalization is straightforward [29], but it involves the Jackson integrals, which have no yet a straightforward generalization to the generic Kerov deformations.

- One could think that (40)-(44) are the only properties of the Schur functions which get significantly changed (into much "heavier" formulas) under the Kerov/Macdonald deformation, but we have found another one. As already mentioned at the beginning of this paper, the definition of Schur functions in the composite representations also appears to be deformed not straightforwardly. An explanation of this fact and the ways to overcome the problem will be a starting point of our far-more-speculative consideration in the next paper [30]. 


\section{Measure $g$-variables as the new times}

As will be illustrated by examples in the Appendix, values of the function $g_{n}$ can actually be treated as new time-variables so that the Kerov functions can be efficiently expressed through the Schur functions of these variables. Moreover, this expansion is somewhat different for the diagrams, which appear at the beginning and at the end of the lexicographical ordering, and the relation between the two expansions is not fully exhausted by (13): there are additional symmetries between the structures for the $k$-th diagram at the beginning and the $k-1$-th one at the end. Already from (13) is follows that if the Schur functions of $p_{k}$ and $g_{k}$ appear in one expansion, they become functions of $p_{k}^{\vee}=p_{k} / g_{k}$ in another, and it appears that $g_{k}$ is also substituted by $g_{k}^{-1}$. The peculiarity of this part of the story is that the expansion in $p_{k}^{\vee}$ is much simpler than the one in $p_{k}$, which is originally used in (11). This expansion is upper instead of lower triangle and, in the Macdonald case, its coefficients are known to be rather simple minors 31. It looks like this result is extendable to the Kerov functions: the coefficients can be expressed through the Schur functions at $p_{k}=g_{k}^{-1}$.

Note that, while $g_{k}$ can clearly be treated as new (additional) time-variables, they participate in additional operations like inversion and multiplication, which are unusual for time-variables. In particular, these operations are not seen on the Miwa loci, and thus are not captured by the usual symmetric functions technique; this can be the reason why these interesting structures were not noticed, and thus theory of the Kerov functions remained underestimated and nearly undeveloped.

- The Kerov functions are polynomials in $p$, but as functions of $g$ they are rational, with $g$-dependent denominators, which are related through (13) to the norms of Kerov functions for transposed diagrams,

$$
\operatorname{Ker}_{R}^{(g)}\{p\}=\frac{\operatorname{pol}_{R}(p, g)}{\Delta_{R}\{g\}}
$$

- For the first diagram in the lexicographical ordering, the Kerov function is independent of $g$, and the denominator is unity:

$$
\operatorname{Ker}_{\left[1^{r}\right]}^{(g)}\{p\}=\operatorname{Schur}_{\left[1^{r}\right]}\{p\}, \quad \Delta_{r}^{(1)}:=\Delta_{\left[1^{r}\right]}=1
$$

- However, already for the second diagram $\left[2,1^{r-2}\right]$ (associated with the adjoint representation of $S L_{r}$ ) there is a non-trivial Macdonald-Kostka coefficient, which is actually the ratio of two Schur functions of $g$-variables:

$$
\mathcal{K}_{\left[2,1^{r-2}\right],\left[1^{r}\right]}^{(g)}=-\frac{\operatorname{Schur}_{[r-1,1]}\{g\}}{\operatorname{Schur}_{[r]}\{g\}}
$$

This implies that

$$
\operatorname{Ker}_{\left[2,1^{r-2}\right]}^{(g)}\{p\}=\operatorname{Schur}_{\left[2,1^{r-2}\right]}\{p\}-\frac{\operatorname{Schur}_{[r-1,1]}\{g\}}{\operatorname{Schur}_{[r]}\{g\}} \cdot \operatorname{Schur}_{\left[1^{r}\right]}\{p\}
$$

Thus, the next denominator is

$$
\Delta_{r}^{(2)}:=\Delta_{\left[2,1^{r-2}\right]}=\operatorname{Schur}_{[r]}\{g\}
$$

If multiplied by $r$ ! it is a polynomial with integer coefficients.

- In the case of the third diagram $\left[2,2,1^{r-2}\right]$, the denominator is described by a somewhat strange 3 -term expression:

$$
\Delta_{r}^{(3)}:=\Delta_{\left[2,2,1^{r-2}\right]}=\operatorname{det}\left(\begin{array}{ccc}
\operatorname{Schur}_{[r]} & 0 & \operatorname{Schur}_{[1]} \\
\operatorname{Schur}_{[r]} & \operatorname{Schur}_{[r-1]} & 0 \\
\operatorname{Schur}_{[r-1]} & \operatorname{Schur}_{[r-2]} & 1
\end{array}\right)
$$


This time it becomes an integer polynomial after multiplication by $r !(r-2)$ !. It appears in similarly looking formulas for the Macdonald-Kostka coefficients

$$
\begin{gathered}
\mathcal{K}_{\left[2,2,1^{r-4}\right],\left[1^{r}\right]}^{(g)}=-\frac{\operatorname{Schur}_{[r-1,1]} \cdot \operatorname{Schur}_{[r-1]}-\operatorname{Schur}_{[r-1,1]} \cdot \operatorname{Schur}_{[r-3]} \cdot \operatorname{Schur}_{[2]}+\operatorname{Schur}_{[r-2,2]} \cdot \operatorname{Schur}_{[r-2]} \cdot \operatorname{Schur}_{[1]}}{\Delta_{r}^{(3)}} \\
\mathcal{K}_{\left[2,2,1^{r-4}\right],\left[2,1^{r-2}\right]}^{(g)}=-\frac{\operatorname{Schur}_{[r-1,1]} \cdot \operatorname{Schur}_{[r-1]}-\operatorname{Schur}_{[r-1,1]} \cdot \operatorname{Schur}_{[r-3]} \cdot \operatorname{Schur}_{[2]}+\operatorname{Schur}_{[r-3,1]} \cdot \operatorname{Schur}_{[r-1]} \cdot \operatorname{Schur}_{[2]}}{\Delta_{r}^{(3)}}
\end{gathered}
$$

and, as a corollary, for the third Kerov function $\operatorname{Ker}_{\left[2,2,1^{r-4}\right]}^{(g)}$. Note that the two functions in (51) differ only in their last terms, the first two are the same.

- The truly interesting is the fourth diagram, $\left[2,2,2,1^{r-6}\right]$, because it is the first one where the two orderings become different: $\left[2,2,2,1^{r-6}\right]<\left[3,1^{r-6}\right]$ but also $\left[2,2,2,1^{r-6}\right]^{\vee}=[r-3,3]<[r-2,1,1]=\left[3,1^{r-6}\right]^{\vee}$; in other words, $\left[2,2,2,1^{r-6}\right]$ is the fourth in the lexicographical ordering, but in transposed ordering the fourth diagram is $\left[3,1^{r-6}\right]$. To get an insight of what to expect from $\Delta^{(4)}$ relevant in this case, it is practical to consider a complementary part of the story, what we will do next. We return to $\Delta^{(4)}$ in (588) below.

- As one can anticipate from (13), the Kerov function in symmetric representation, which is the last in the lexicographical ordering, is almost as simple as in the antisymmetric one, which is the first, despite this is not easy to see directly from the definition (11). In fact, (13) implies that $\operatorname{Ker}_{[r]}$ is a Schur polynomial of $p_{k} / g_{k}$, but the $g$-dependence of the prefactor still needs to be found. In this case, it is easy. According to (13), what is needed is the norm of the antisymmetric Schur function:

$$
\begin{aligned}
& \operatorname{Ker}_{[r]}^{(g)}\{p\} \stackrel{\underline{13}}{=} \frac{(-)^{|R|} \operatorname{Ker}_{\left[1^{r}\right]}^{\left(g^{-1}\right)}\left\{-p_{k} / g_{k}\right\}}{\left\|\operatorname{Ker}_{\left[1^{r}\right]}^{\left(g^{-1}\right)}\right\|^{2}}=\frac{(-)^{|R|} \operatorname{Schur}_{\left[1^{r}\right]}\left\{-p_{k} / g_{k}\right\}}{\left\|\operatorname{Ker}_{\left[1^{r}\right]}^{\left(g^{-1}\right)}\right\|^{2}}=\frac{\operatorname{Schur}_{[r]}\left\{p_{k} / g_{k}\right\}}{\left\|\operatorname{Ker}_{\left[1^{r}\right]}^{\left(g^{-1}\right)}\right\|^{2}} \Longrightarrow \\
& \Delta_{r}^{\vee} \sim\left\|\operatorname{Ker}_{\left[1^{r}\right]}^{\left(g^{-1}\right)}\right\|^{2}=\left\langle\operatorname{Schur}_{\left[1^{r}\right]} \mid \operatorname{Schur}_{\left[1^{r}\right]}\right\rangle^{\left(g^{-1}\right)} \stackrel{41]+5}{=} \sum_{\Delta \vdash r} \frac{\psi_{\left[1^{r}\right]}(\Delta)^{2}}{z_{\Delta}} \frac{1}{g^{\Delta}}=\operatorname{Schur}_{[r]}\left\{g_{k}^{-1}\right\}
\end{aligned}
$$

where $g^{\Delta}=\prod g_{\delta_{i}}$, and, at the last stage, we used peculiarities of antisymmetric and symmetric representations: $\psi_{\left[1^{r}\right]}(\Delta)=(-)^{l_{R}+|R|}$ and $\psi_{[r]}(\Delta)=1$ for any $\Delta$ of the size $r$. $l_{R}$ Thus, for the Kerov functions in symmetric representations, we get a universal expression

$$
\operatorname{Ker}_{[r]}^{(g)}\{p\}=\frac{\operatorname{Schur}_{[r]}\left\{\frac{p_{k}}{g_{k}}\right\}}{\operatorname{Schur}_{[r]}\left\{\frac{1}{g_{k}}\right\}}
$$

explicitly realizing their relation (13) to the $g$-independent Kerov functions in antisymmetric representations

$$
\operatorname{Ker}_{\left[1^{r}\right]}^{(g)}\{p\}=\operatorname{Schur}_{\left[1^{r}\right]}\left\{p_{k}\right\}
$$

- Remarkably, the denominator in (53), which has no counterpart in (54), is related to the one in (48):

$$
\Delta_{[r]}\left\{g_{k}\right\}=\Delta_{r}^{(2)}\left\{g_{k}^{-1}\right\}
$$

- Moreover, this property persists further: the denominator in the case of penultimate (second from the end) diagram $[r-1,1]$ is obtained by $g$-inversion from that for the third one, $\left[2,2,1^{r-2}\right]$ :

$$
\Delta_{[r-1,1]}\left\{g_{k}\right\}=\Delta_{r}^{(3)}\left\{g_{k}^{-1}\right\}
$$

Given (50), this is an absolutely explicit formula, and it enters the equally explicit expression for the corresponding Kerov function:

$$
\operatorname{Ker}_{[r-1,1]}^{(g)}\{p\}=\frac{\operatorname{Schur}_{[r]}\left\{\frac{1}{g_{k}}\right\} \cdot \operatorname{Schur}_{[r-1,1]}\left\{\frac{p_{k}}{g_{k}}\right\}-\operatorname{Schur}_{[r-1,1]}\left\{\frac{1}{g_{k}}\right\} \cdot \operatorname{Schur}_{[r]}\left\{\frac{p_{k}}{g_{k}}\right\}}{\left(\operatorname{Schur}_{[1]} \cdot \Delta_{r}^{(3)}\right)\left\{\frac{1}{g_{k}}\right\}}
$$


Note that, while the denominator is related to that for $\operatorname{Ker}_{\left[2,2,1^{r-4}\right]}^{(g)}$ (this is not quite the relation (13), the two diagrams are not transposed, but additionally shifted by one in the lexicographical ordering!), this is not immediately so for the numerators. Instead, the numerators of the Kerov functions in the vicinity of symmetric case demonstrate a structure of minor expansion, which should be a straightforward generalization of the one found in 31] for the Macdonald functions.

- Since for the particular value of $r=3$, the first diagram from the end coincides with the third one from the beginning, we can expect that, in this case, $\Delta^{(2)}\left\{g^{-1}\right\}$ when expanded in $g$ will have the same shape as $\Delta^{(3)}$, i.e. the Schur function of inverted $g$ will be a three-term determinant. This is indeed the case, and it implies that the shape of $\Delta_{[r-2,2]}$ can actually reveal the structure of $\Delta^{(4)}\left\{g^{-1}\right\}$ and thus of $\Delta^{(4)}\{g\}$. Already the simplest $\Delta_{[2,2]}$ is given by a somewhat sophisticated 19-term formula (see the very end of the next section), and this illustrates the type of problems one should deal with and resolve.

- The full expression for $\Delta^{(4)}$ is rather lengthy, for illustrative purposes, we present just the first three terms, which fully capture the contribution with the product $g_{[r]} g_{[r-1]}$ :

$$
\left.\begin{array}{c}
\Delta_{r}^{(4)}:=\Delta_{\left[2,2,2,1^{r-6}\right]} \\
\widehat{\Delta}_{r}^{(4)}:=\widehat{\Delta}_{\left[3,1^{r-3}\right]}
\end{array}\right\}=
$$

(for example of the full expression in a particular case, see the last formula in (71) in the Appendix). This time the factorial would be $((r-2) !)^{2}(r-4)$ !. If so normalized, $\Delta_{r}^{(4)}$ at even $r$ are divisible by 2 (i.e. are even integer polynomials). What is important, the hatted and ordinary quantities are the same,

$$
\Delta^{(4)}=\widehat{\Delta^{(4)}}
$$

despite they appear in the denominators of very different functions, $\operatorname{Ker}_{\left[2,2,2,1^{r-6}\right]}$ and $\widehat{\operatorname{Ker}}_{\left[3,1^{r-3}\right]}$. It is interesting, if this property (transposition independence) persists for all higher $\Delta^{(m)}$.

- We emphasize that all these strangely looking combinations of the Schur functions in $\Delta$ 's are factorized at the topological locus, which explains factorization of the associated Macdonald quantities. The Macdonald factorizations begin to attract interest in various contexts, see, for example, [12,32, 33, and our observations suggest that these studies can have relation to formulas for the Kerov functions (which themselves are not factorized). Factorizations are also crucial for knot theory applications, and further extension of, say, the Rosso-Jones formula, which defines the Macdonald based torus knot/link hyperpolynomials, to the Kerov functions depends on understanding of what substitutes the factorization in the Kerov case.

\section{Conclusion}

To conclude, we presented a list of properties of the Kerov functions. Our goal was to illustrate that theory of these functions is very rich, and they rather have more interesting properties that the Schur and Macdonald functions, in contrary to the common belief. Lost in the Kerov case are very special features like vanishing the multiplication structure constants for diagrams which are not in the product of representations and similar rigid (precise) links with representation theory, as well as a Sugawara-like construction of the generalized cut-and-join operators through the $U(1)$-like Cherednik-Dunkl operators. Though important, these properties are not truly crucial for many applications, especially because the other ones, which are really needed for practical calculations, like the Cauchy summation formula (19) for the skew Kerov functions and even the transposition rule (13) nicely persist. Instead of the lost properties, an entire new world of additional timevariables $g_{k}$ emerges. Moreover, a role appears for non-trivial operations over the time-variable like inversion and multiplication, and these unexpected operations do act on the Kerov functions, quite non-trivially, but nicely and explicitly.

We hope that our modest review will attract an attention to this interesting field. Particular applications were mentioned in the introduction, they will be addressed elsewhere. A special role among them is played by knot theory applications, also because this theory provides one of the most promising approaches to quantum programming [34,35. 


\section{Acknowledgements}

Our original interest to Kerov functions was largely inspired by Anton Zabrodin many years ago. We are indebted to Anton Khoroshkin for fresh comments on existing folklore about the role and (in)significance of dominance rule and other peculiarities of Macdonald theory. We highly appreciate our constant discussions on related subjects with Hidetoshi Awata, Hiroaki Kanno, Andrey Morozov, Alexei Sleptsov and Yegor Zenkevich.

This work was supported by Russian Science Foundation grant No 18-71-10073.

\section{Appendix. Examples}

Kerov functions $\widehat{\text { Ker }}$ and Ker begin to deviate from each other only at level $|R|=6$, which is beyond this example section, thus we do not distinguish between them here. At various smaller levels, we illustrate other important phenomena.

\subsection{Level 1}

Like $\operatorname{Ker}_{\emptyset}=1$ at level $|R|=0$, the polynomial at the first level is also universal, but already its norm is g-dependent:

$$
\operatorname{Ker}_{[1]}^{(g)}=p_{1}, \quad\left\|\operatorname{Ker}_{[1]}^{(g)}\right\|^{2}=g_{1}, \quad \operatorname{Ker}_{[1]}\{p\}=p_{1} \stackrel{13}{=}\left\|\operatorname{Ker}_{[1]}^{(g)}\right\|^{2} \cdot \operatorname{Ker}_{[1]}\left\{-\frac{p_{k}}{g_{k}}\right\}
$$

In what follows, we omit the index $(g)$ to simplify the formulas.

\subsection{Level 2}

At level 2, there are just two functions

$$
\begin{array}{r}
\operatorname{Ker}_{[1,1]}=\operatorname{Schur}_{[1,1]}=\frac{-p_{2}+p_{1}^{2}}{2} \\
\operatorname{Ker}_{[2]}=\operatorname{Schur}_{[2]}+K \cdot \operatorname{Schur}_{[1,1]}=\frac{(1-K) p_{2}+(1+K) p_{1}^{2}}{2}=\frac{g_{1}^{2} p_{2}+g_{2} p_{1}^{2}}{g_{2}+g_{1}^{2}}=\frac{2 g_{2} g_{1}^{2}}{g_{2}+g_{1}^{2}} \cdot \operatorname{Schur}_{[2]}\left\{-\frac{p_{k}}{g_{k}}\right\}
\end{array}
$$

with the norms $\left\|\operatorname{Ker}_{[1,1]}\right\|^{2}=\frac{g_{2}+g_{1}^{2}}{2}$ and $\left\|\operatorname{Ker}_{[2]}\right\|^{2}=\frac{2 g_{2} g_{1}^{2}}{g_{2}+g_{1}^{2}}$. Conditions (13) imply that

$$
\begin{array}{r}
\operatorname{Ker}_{[2]}\{p\}=\left\|\operatorname{Ker}_{[2]}\right\|^{2} \cdot \operatorname{Ker}_{[1,1]}^{\vee}\left\{-p^{\vee}\right\} \quad \Longleftrightarrow \frac{g_{1}^{2} p_{2}+g_{2} p_{1}^{2}}{g_{2}+g_{1}^{2}}=\frac{2 g_{1}^{2} g_{2}}{g_{2}+g_{1}^{2}} \cdot \frac{p_{2}^{\vee}+p_{1}^{\vee}}{2} \\
\operatorname{Ker}_{[1,1]}\{p\}=\left\|\operatorname{Ker}_{[1,1]}\right\|^{2} \cdot \operatorname{Ker}_{[2]}^{\vee}\left\{-p^{\vee}\right\} \quad \Longleftrightarrow \quad \frac{-p_{2}+p_{1}^{2}}{2}=\frac{-g_{1}^{\vee} p_{2}^{\vee}+g_{2}^{\vee} p_{1}^{\vee}}{2} \cdot \frac{g_{1}^{2}+g_{2}^{2}}{g_{1}^{\vee 2}+g_{2}^{\vee}}
\end{array}
$$

which can be easily solved:

$$
p_{2}^{\vee}=\frac{p_{2}}{g_{2}}, \quad p_{1}^{\vee}=\frac{p_{1}}{g_{1}}, \quad g_{1}^{\vee}=\frac{1}{g_{1}}, \quad g_{2}^{\vee}=\frac{1}{g_{2}}
$$

The product (10)

$$
\operatorname{Ker}_{[1]}^{2}=\operatorname{Ker}_{[2]}+\frac{2 g_{1}^{2}}{g_{2}+g_{1}^{2}} \cdot \operatorname{Ker}_{[1,1]} \quad \Longrightarrow \quad N_{[1],[1]}^{[2]}(g)=1, \quad N_{[1],[1]}^{[1,1]}(g)=\frac{2 g_{1}^{2}}{g_{2}+g_{1}^{2}}=\frac{S_{[1]}^{2}}{S_{[2]}}
$$

is related to the shape of the skew Kerov functions:

$$
\operatorname{Ker}_{[2] /[1]}=\frac{2 g_{2}}{g_{2}+g_{1}^{2}} \cdot \operatorname{Ker}_{[1]}=N_{[1],[1]}^{[2]^{\vee}}\left(g^{-1}\right) \cdot \operatorname{Ker}_{[1]}, \quad \operatorname{Ker}_{[11] /[1]}=\operatorname{Ker}_{[1]}=N_{[1],[1]}^{[1,1]^{\vee}} \cdot \operatorname{Ker}_{[1]}
$$




\subsection{Level 3}

This is the first level where different $g$-dependent denominators emerge in the formulas, but they are still related by the $g$-inversion:

$$
\begin{array}{r}
\operatorname{Ker}_{[3]}=\frac{2 g_{2} g_{1}^{3} p_{3}+3 g_{3} g_{1}^{2} p_{2} p_{1}+g_{3} g_{2} p_{1}^{3}}{2 g_{2} g_{1}^{2}+3 g_{3} g_{1}^{2}+g_{3} g_{2}}=\frac{\operatorname{pol}(p, g)}{\Delta_{3}^{\vee}}=\frac{\operatorname{Schur}_{[3]}\left\{\frac{p_{k}}{g_{k}}\right\}}{\operatorname{Schur}_{[3]}\left\{\frac{1}{g_{k}}\right\}} \\
\operatorname{Ker}_{[2,1]}=\frac{-g_{1}\left(g_{2}+g_{1}^{2}\right) p_{3}+\left(g_{1}^{3}-g_{3}\right) p_{2} p_{1}+\left(g_{3}+g_{2} g_{1}\right) p_{1}^{3}}{2 g_{3}+3 g_{2} g_{1}+g_{1}^{3}}=\frac{\operatorname{pol}(p, g)}{\Delta_{3}} \\
\operatorname{Ker}_{[1,1,1]}=\operatorname{Schur}_{[1,1,1]}=\frac{p_{3}}{3}-\frac{p_{2} p_{1}}{2}+\frac{p_{1}^{3}}{6}
\end{array}
$$

where the denominators are related by the inversion of $g: \Delta_{3}^{\vee}\{g\}:=2 g_{2} g_{1}^{2}+3 g_{3} g_{1}^{2}+g_{3} g_{2}=g_{3} g_{2} g_{1}^{3} \cdot \Delta_{3}\left\{g^{-1}\right\}$ with $\Delta_{3}\{g\}=2 g_{3}+3 g_{2} g_{1}+g_{1}^{3}=6 \operatorname{Schur}_{[3]}\left\{g_{1}, g_{2}, g_{3}\right\}$.

The products and skew Kerov functions are

$$
\begin{gathered}
\operatorname{Ker}_{[2]} \cdot \operatorname{Ker}_{[1]}=\operatorname{Ker}_{[3]}+ \\
+\frac{2 g_{2} g_{1}^{2}\left(2 g_{3}+3 g_{2} g_{1}+g_{1}^{3}\right)}{\left(g_{2}+g_{1}^{2}\right)\left(g_{3} g_{2}+3 g_{3} g_{1}^{2}+2 g_{2} g_{1}^{3}\right)} \cdot \operatorname{Ker}_{[2,1]}
\end{gathered} \quad\left\{\begin{array}{c}
N_{[2],[1]}^{[3]}(g)=1 \\
\operatorname{Ker}_{[1,1]} \cdot \operatorname{Ker}_{[1]}=\operatorname{Ker}_{[2,1]}+\frac{3 g_{1}\left(g_{2}+g_{1}^{2}\right)}{2 g_{3}+3 g_{2} g_{1}+g_{1}^{3}} \cdot \operatorname{Ker}_{[1,1,1]} \\
N_{[2],[1]}^{[2,1]}(g)=\frac{2 g_{2} g_{1}^{2}\left(2 g_{3}+3 g_{2} g_{1}+g_{1}^{3}\right)}{\left(g_{2}+g_{1}^{2}\right)\left(g_{3} g_{2}+3 g_{3} g_{1}^{2}+2 g_{2} g_{1}^{3}\right)} \\
N_{[1,1],[1]}^{[2,1]}(g)=1 \\
N_{[1,1],[1]}^{[1,1,1]}(g)=\frac{3 g_{1}\left(g_{2}+g_{1}^{2}\right)}{2 g_{3}+3 g_{2} g_{1}+g_{1}^{3}}=\frac{\operatorname{Schur}_{[2]}\{g\} \cdot \operatorname{Schur}[1]\{g\}}{\operatorname{Schur}[3]\{g\}}
\end{array}\right.
$$

$$
\operatorname{Ker}_{[3] /[2]}=N_{[1,1],[1]}^{[1,1,1]}\left(g^{-1}\right) \cdot \operatorname{Ker}_{[1]}, \quad \operatorname{Ker}_{[3] /[1]}=N_{[1,1],[1]}^{[1,1,1]}\left(g^{-1}\right) \cdot \operatorname{Ker}_{[2]}
$$

$$
\operatorname{Ker}_{[2,1] /[2]}=N_{[1,1],[1]}^{[2,1]}\left(g^{-1}\right) \cdot \operatorname{Ker}_{[1]}, \quad \operatorname{Ker}_{[2,1] /[1,1]}=N_{[2],[1]}^{[2,1]}\left(g^{-1}\right) \cdot \operatorname{Ker}_{[1]}=\operatorname{Ker}_{[1]},
$$

$$
\begin{array}{r}
\operatorname{Ker}_{[2,1] /[1]}=N_{[1,1],[1]}^{[2,1]}\left(g^{-1}\right) \cdot \operatorname{Ker}_{[2]}+N_{[2],[1]}^{[2,1]}\left(g^{-1}\right) \cdot \operatorname{Ker}_{[1,1]}=\operatorname{Ker}_{[2]}+N_{[2],[1]}^{[2,1]}\left(g^{-1}\right) \cdot \operatorname{Ker}_{[1,1]} \\
\operatorname{Ker}_{[1,1,1] /[1,1]}=N_{[2],[1]}^{[3]}\left(g^{-1}\right) \cdot \operatorname{Ker}_{[1]}=\operatorname{Ker}_{[1]}, \quad \operatorname{Ker}_{[1,1,1] /[1]}=N_{[2],[1]}^{[3]}\left(g^{-1}\right) \cdot \operatorname{Ker}_{[1,1]}=\operatorname{Ker}_{[2]}
\end{array}
$$

Note also that

$$
\Delta_{3} \cdot \operatorname{Ker}_{[2,1]}=-g_{1} p_{3} \cdot \Delta_{2}+g_{1} p_{1} \cdot \Delta_{2} \cdot \operatorname{Ker}_{[2]}+2 g_{3} p_{1} \cdot \operatorname{Ker}_{[1,1]}
$$

\subsection{Level 4}

Expressions for various denominators $\Delta_{4}\{g\}$ will be provided in the next subsection. Other quantities are

$$
\begin{array}{r}
\operatorname{Ker}_{[4]}=\frac{6 g_{3} g_{2}^{2} g_{1}^{4} \cdot p_{4}+8 g_{4} g_{2}^{2} g_{1}^{3} \cdot p_{3} p_{1}+3 g_{4} g_{3} g_{1}^{4} \cdot p_{2}^{2}+6 g_{4} g_{3} g_{2} g_{1}^{2} \cdot p_{2} p_{1}+g_{4} g_{3} g_{2}^{2} \cdot p_{1}^{4}}{\Delta_{4}^{\vee}}=\frac{\operatorname{Schur}_{[4]}\left\{\frac{p_{k}}{g_{k}}\right\}}{\operatorname{Schur}_{[4]}\left\{\frac{1}{g_{k}}\right\}} \\
\operatorname{Ker}_{[3,1]}=\frac{\operatorname{pol}(p, g)}{2 \Delta_{4}^{\prime \vee}}, \quad \operatorname{Ker}_{[2,2]}=\frac{\operatorname{pol}(p, g)}{2 \Delta_{4}^{\prime}}, \quad \operatorname{Ker}_{[1,1,2]}=\frac{\operatorname{pol}(p, g)}{2 \Delta_{4}} \\
\operatorname{Ker}_{[1,1,1,1]}=\operatorname{Schur}_{[1,1,1,1]}\left\{p_{k}\right\}
\end{array}
$$

The simplest of the three polynomials in the numerators is in

$\operatorname{Ker}_{[1,1,2]}=\frac{g_{1} \overbrace{\left(2 g_{3}+3 g_{2} g_{1}+g_{1}^{3}\right)}^{\Delta_{3}} \cdot\left(2 p_{4}-p_{2}^{2}\right)+2\left(2 g_{4}+g_{2}^{2}-2 g_{2} g_{1}^{2}-g_{1}^{4}\right) \cdot p_{3} p_{1}-\left(6 g_{4}+4 g_{3} g_{1}+3 g_{2}^{2}-g_{1}^{4}\right) \cdot p_{2} p_{1}^{2}+\left(2 g_{4}+2 g_{3} g_{1}+g_{2}^{2}+g_{2} g_{1}^{2}\right) \cdot p_{1}^{4}}{2 \Delta_{4}}$

but they are hardly useful, if presented in this form. Remarkably, this complicated expression is nothing but a very simple (48), which is a direct generalization of (69). 


\subsection{Examples of the emerging structure}

We can now list the emerging denominators and observe that they are actually the Schur functions with $g_{k}$ playing the role of the time variables:

$$
\begin{aligned}
& \Delta_{2}=g_{2}+g_{1}^{2}=2 \operatorname{Schur}_{[2]}\left\{g_{1}, g_{2}\right\} \\
& \Delta_{3}=2 g_{3}+3 g_{2} g_{1}+g_{1}^{3}=6 \operatorname{Schur}_{[3]}\left\{g_{1}, g_{2}, g_{3}\right\} \text {, } \\
& \Delta_{3}^{\vee}=g_{3} g_{2}+3 g_{3} g_{1}^{2}+2 g_{2} g_{1}^{3}=6 g_{3} g_{2} g_{1}^{3} \cdot \operatorname{Schur}_{[3]}\left(g_{1}^{-1}, g_{2}^{-1}, g_{3}^{-1}\right)= \\
& =6\left(\operatorname{Schur}_{[3]} \cdot \operatorname{Schur}_{[2]}+\operatorname{Schur}_{[3]} \cdot \operatorname{Schur}_{[1]}^{2}-\operatorname{Schur}_{[2]}^{2} \cdot \operatorname{Schur}_{[1]}\right)\{g\} \sim \\
& \sim([32]+[311]-[221])=\operatorname{det}\left(\begin{array}{ccc}
\operatorname{Schur}_{[3]} & 0 & \operatorname{Schur}_{[1]} \\
\operatorname{Schur}_{[3]} & \operatorname{Schur}_{[2]} & 0 \\
\operatorname{Schur}_{[2]} & \operatorname{Schur}_{[1]} & 1
\end{array}\right) \\
& \Delta_{4}=6 g_{4}+8 g_{3} g_{1}+3 g_{2}^{2}+6 g_{2} g_{1}^{2}+g_{1}^{4}=24 \operatorname{Schur}_{[4]}\left\{g_{k}\right\} \\
& \Delta_{4}^{\vee}=g_{4} g_{3} g_{2}^{2}+6 g_{4} g_{3} g_{2} g_{1}^{2}+8 g_{4} g_{2} g_{1}^{2}+3 g_{4} g_{3} g_{1}^{4}+6 g_{3} g_{2}^{2} g_{1}^{4} \sim \operatorname{Schur}_{[4]}\left\{g_{k}^{-1}\right\} \\
& \Delta_{4}^{\prime}=2\left(2 g_{4} g_{3}+g_{3} g_{2}^{2}+6 g_{4} g_{2} g_{1}+3 g_{2}^{3} g_{1}+2 g_{3} g_{2} g_{1}^{2}+4 g_{4} g_{1}^{3}+2 g_{2}^{2} g_{1}^{3}+3 g_{3} g_{1}^{4}+g_{2} g_{1}^{5}\right)= \\
& =48\left(\operatorname{Schur}_{[4]} \cdot \operatorname{Schur}_{[3]}+\operatorname{Schur}_{[4]} \cdot \operatorname{Schur}_{[2]} \cdot \operatorname{Schur}_{[1]}-\operatorname{Schur}_{[3]}^{2} \cdot \operatorname{Schur}_{[1]}\right)\{g\} \sim \\
& \sim([43]+[421]-[331])=\operatorname{det}\left(\begin{array}{ccc}
\operatorname{Schur}_{[4]} & 0 & \operatorname{Schur}_{[1]} \\
\operatorname{Schur}_{[4]} & \operatorname{Schur}_{[3]} & 0 \\
\operatorname{Schur}_{[3]} & \operatorname{Schur}_{[2]} & 1
\end{array}\right) \\
& \Delta_{4}^{\prime \vee}=\Delta_{4}^{\prime}\left\{g_{k}^{-1}\right\} \sim(\underline{2[4322]+[431111]}+2[42221]-4[422111]+[4211111]- \\
& -2[33221]-[3311111]-[32222]+4[322211]-[222221])
\end{aligned}
$$

Explicitly written in (58) is the lifting to arbitrary $r$ of just the two underlined terms from the last formula. Note that the degeneracy is lifted for $r>4$, and they become three independent structures. The same is going to happen to the other items, thus, in general, it is a 19-term expression.

This form of $\Delta_{r}$ can explain why the Macdonald choice of $g_{k}$ is distinguished: it is an exact counterpart of the topological locus, which converts the Schur functions into the quantum dimensions $g_{k}=\frac{\left\{A^{k}\right\}}{\left\{t^{k}\right\}}$. However, the factorization of other denominators is far from obvious. What if we further change the Schur functions of $g_{k}$ for the Macdonald ones and then to the Kerov functions?

Since this Appendix is devoted to honest examples, which illustrate and underlie general theory rather than follow from it, we do not interpret the denominators $\Delta$ in the terms of sec 7 instead we present them as they are. Actually, in general terms (i.e. for all $r$ ),

$$
\begin{array}{cc}
\begin{array}{c}
\text { second diagram } \\
\text { third diagram } \\
\text { fourth diagram }
\end{array} & \Delta_{r}=\Delta_{\left[2,1^{r-2}\right]}=\Delta_{r}^{(2)}\left\{g_{k}\right\} \\
\ldots & \Delta_{r}^{\prime}=\Delta_{\left[2,2,1^{r-2}\right]}=\Delta_{r}^{(3)}\left\{g_{k}\right\} \\
\text { third-from-the end diagram } & \Delta_{\left[2,2,2,1^{r-6}\right]}=\widehat{\Delta}_{\left[3,1^{r-3}\right]}=\Delta_{r}^{(4)}\left\{g_{k}\right\} \\
\text { penultimate diagram } & \Delta_{r}^{\prime \prime \vee}=\Delta_{[r-2,2]}=\Delta_{r}^{\prime \prime}\left\{g_{k}^{-1}\right\} \\
\text { last diagram } & \Delta_{r}^{\prime \vee}=\Delta_{[r-1,1]}=\Delta_{r}^{\prime}\left\{g_{k}^{-1}\right\} \\
& \Delta_{r}^{\vee}=\Delta_{[r]}=\Delta_{r}\left\{g_{k}^{-1}\right\}
\end{array}
$$


Note that the lexicographically-last diagram is related by the $g$-inversion to the second diagram, not to the first one, and so on: there is a shift-by-one in these formulas as compared to a more naive expectation from (13). In result, while the "true" fourth diagram appears only at level 6 , its counterpart is the third diagram from the end, and it is non-trivial already at level 4 , which allows one to learn something about $\Delta^{(4)}$ from the example at this level. The structure which is revealed in this way is indeed true in general, see (58) in sec 7

\section{References}

[1] H.Awata, H.Kanno, A.Mironov, A.Morozov, An.Morozov, Phys.Rev. D98 (2018) 046018, arXiv:1806.01146

[2] J.W. Alexander, Trans.Amer.Math.Soc. 30 (2) (1928) 275-306

V.F.R.Jones, Invent.Math. 72 (1983) 1; Bull.AMS 12 (1985) 103; Ann.Math. 126 (1987) 335

L. Kauffman, Topology 26 (1987) 395

P.Freyd, D.Yetter, J.Hoste, W.B.R.Lickorish, K.Millet, A.Ocneanu, Bull. AMS. 12 (1985) 239

J.H. Przytycki, K.P. Traczyk, Kobe J Math. 4 (1987) 115-139

[3] M. Aganagic, M. Mariño, C. Vafa, Comm.Math.Phys. 247 (2004) 467-512, hep-th/0206164

M. Aganagic, A. Klemm, M. Marino, C. Vafa, Comm.Math.Phys. 254 (2005) 425, hep-th/0305132

[4] H. Awata et al., to appear

[5] S.V. Kerov, Func.An.and Apps. 25 (1991) 78-81

In variance with "the Kerov polynomials", the literature on these Kerov functions is quite limited, e.g. see 6]

[6] T.H. Baker, Symmetric functions and infinite-dimensional algebras, PhD Thesis, 1994, Tasmania

A.H. Bougourzi, L. Vinet, Letters in Mathematical Physics 39 (1997) 299311, q-alg/9604021

A.A. Bytsenko, M. Chaichian, R.J. Szabo, A. Tureanu, arXiv:1308.2177

A.A. Bytsenko, M. Chaichian, R. Luna, J.Math.Phys. 58 (2017) 121701, arXiv:1707.01553

[7] P. Biane, in: Asymptotic Combinatorics with Applications to Mathematical Physics, A. Vershik (Ed.), Springer Lecture Notes in Mathematics 1815 (2003) 185-200

[8] H. Awata, B. Feigin, A. Hoshino, M. Kanai, J. Shiraishi, S. Yanagida, arXiv:1106.4088

A. Morozov, A. Smirnov, Lett.Math.Phys. 104 (2014) 585, arXiv:1307.2576

S. Mironov, An. Morozov, Y. Zenkevich, JETP Lett. 99 (2014) 109, arXiv:1312.5732

Y. Ohkubo, arXiv:1404.5401

B. Feigin, M. Jimbo, T. Miwa, E. Mukhin, arXiv:1502.07194

[9] Y. Zenkevich, arXiv:1712.10300

A. Morozov, Phys.Lett. B785 (2018) 175-183, arXiv:1808.01059; $\operatorname{arXiv:1810.00395}$

[10] I.G. Macdonald, Symmetric functions and Hall polynomials, Second Edition, Oxford University Press, 1995

[11] P.G.O. Freund, A.V. Zabrodin, Phys.Lett. B294 (1992) 347353, hep-th/9208063

[12] P.Dunin-Barkowski, A.Mironov, A.Morozov, A.Sleptsov, A.Smirnov, JHEP 03 (2013) 021, arXiv:1106.4305

[13] A.Mironov, A.Morozov, An.Morozov, AIP Conf. Proc. 1562 (2013) 123, arXiv:1306.3197

[14] N.M. Dunfield, S. Gukov, J. Rasmussen, Experimental Math. 15 (2006) 129-159, math/0505662

H. Itoyama, A. Mironov, A. Morozov, An. Morozov, JHEP 2012 (2012) 131, arXiv:1203.5978

E. Gorsky, S. Gukov, M. Stosic, arXiv:1304.3481

S. Arthamonov, A. Mironov, A. Morozov, An. Morozov, JHEP 04 (2014) 156, arXiv:1309.7984

S. Gukov, S. Nawata, I. Saberi, M. Stosic, P. Sulkowski, JHEP 1603 (2016) 004, arXiv:1512.07883

[15] A. Mironov, A. Morozov, An. Morozov, in: Strings, Gauge Fields, and the Geometry Behind: The Legacy of Maximilian Kreuzer, eds: A.Rebhan, L.Katzarkov, J.Knapp, R.Rashkov, E.Scheidegger, World Scietific, 2013 pp.101-118 arXiv:1112.5754

[16] M. Rosso, V. F. R. Jones, J. Knot Theory Ramifications, 2 (1993) 97-112

M. Tierz, Mod. Phys. Lett. A19 (2004) 1365-1378, hep-th/0212128

A. Brini, B. Eynard, M. Mariño, Annales Henri Poincaré, 13, No. 8, SP Birkhäuser Verlag Basel, 2012, arXiv:1105.2012 
[17] M. Aganagic, Sh. Shakirov, arXiv:1105.5117

[18] I. Cherednik, arXiv:1111.6195

[19] A. Mironov, A. Morozov, An. Morozov, JHEP 1203 (2012) 034, arXiv:1112.2654

[20] R. Howe, Trans. Amer. Math. Soc. 313 (1989), 539-570; J. Amer. Math. Soc. 2 (1989) 535-552

R. Goodman, N.R. Wallach, Representations and Invariants of the Classical Groups, Cambridge University Press, 1998

[21] M. Varagnolo, E. Vasserot, Commun. Math. Phys. 182 (1996) 469483, q-alg/9506026

O. Schiffmann, E. Vasserot, Compositio Mathematica 147 (2011) 188-234, arXiv:0802.4001; Duke Mathematical Journal 162 (2013) 279-366, arXiv:0905.2555, arXiv:1202.2756

[22] J. Ding, K. Iohara, Lett.Math.Phys. 41 (1997) 181-193, q-alg/9608002

K. Miki, J. Math. Phys. 48 (2007) 123520

[23] A. Mironov, A. Morozov, S. Natanzon, Theor.Math.Phys. 166 (2011) 1-22, arXiv:0904.4227; Journal of Geometry and Physics 62 (2012) 148-155, arXiv:1012.0433

[24] V. Knizhnik, A. Zamolodchikov, Nucl. Phys. B247 (1984) 83

[25] J. Wess, B. Zumino, Phys. Lett. B37 (1971) 95

S. Novikov, Usp. Mat. Nauk 37 (1982) 3

E. Witten, Comm. Math. Phys. 92 (1984) 455

A. Polyakov, P. Wiegmann, Phys.Lett. B131 (1983) 121; Phys.Lett. B141 (1984) 223

[26] A. Mironov, A. Morozov, JHEP 2018 (2018) 163, arXiv:1807.02409

[27] A. Mironov, A. Morozov, Phys.Lett. B771 (2017) 503-507, arXiv:1705.00976

[28] A. Mironov, A. Morozov, Phys.Lett. B774 (2017) 210-216, arXiv:1706.03667

H. Itoyama, A. Mironov, A. Morozov, arXiv:1808.07783

[29] H.Awata, Y.Yamada, JHEP 1001 (2010) 125, arXiv:0910.4431; Prog.Theor.Phys. 124 (2010) 227, arXiv: 1004.5122

A. Mironov, A. Morozov, S. Shakirov, A. Smirnov, Nucl. Phys. B855 (2012) 128, arXiv:1105.0948

H. Itoyama, T. Oota, R. Yoshioka, arXiv:1602.01209

A. Morozov, A. Popolitov, Sh. Shakirov, Phys.Lett. B784 (2018) 342-344, arXiv:1803.11401

[30] On Macdonald and Kerov functions for composite representations, in preparation

[31] L. Lapointe, A. Lascoux, J. Morse, arXiv:math/9808050

[32] Y. Kononov, A. Morozov, Eur.Phys.J. C76 (2016) 424, arXiv:1607.00615

Y. Zenkevich, arXiv:1612.09570

[33] L. Colmenarejo, C.F. Dunkl, J.-G. Luque, Symmetry 10 (2018) 541, arXiv:1707.00897

[34] P.K. Aravind, in: Potentiality, Entanglement and Passion-at-a-Distance, ed. by R.S. Cohen et al, pp. 53-59, Kluwer, 1997

L. Kauffman, S. Lomonaco, New Journal of Physics, 4 (2002) 73.1-18; 6 (2004) 134.1-40, quant-ph/0401090

D. Melnikov, A. Mironov, S. Mironov, A. Morozov, An. Morozov, Nucl.Phys. B926 (2018) 491-508, arXiv:1703.00431

[35] V. Balasubramanian, J.R. Fliss, R.G. Leigh, O. Parrikar, JHEP, 2017 (2017) 61, arXiv:1611.05460

V. Balasubramanian, M. DeCross, J. Fliss, A. Kar, R.G. Leigh, O. Parrikar, arXiv:1801.01131

D. Melnikov, A. Mironov, S. Mironov, A. Morozov, An. Morozov, arXiv:1809.04574 